\title{
DiVA
}

http://kth.diva-portal.org

This is an author produced version of a paper published in 7th International Power Electronics and Motion Control Conference (IPEMC), 2012.

This paper has been peer-reviewed but does not include the final publisher proofcorrections or proceedings pagination.

(C) 2012 IEEE. Personal use of this material is permitted. Permission from IEEE must be obtained for all other uses, in any current or future media, including reprinting/republishing this material for advertising or promotional purposes, creating new collective works, for resale or redistribution to servers or lists, or reuse of any copyrighted component of this work in other works.

Citation for the published paper:

Jacek Rabkowski, Dimosthenis Peftitsis, Hans-Peter Nee, Mariusz Zdanowski. A Simple High-Performance Low-Loss Current-Source Driver for SiC Bipolar Transistors.

7th International Power Electronics and Motion Control Conference (IPEMC), 2012

Access to the published version may require subscription.

Published with permission from: IEEE 


\section{A Simple High-Performance Low-Loss Current- Source Driver for SiC Bipolar Transistors}

\author{
Jacek Rabkowski, Dimosthenis Peftitsis, Hans-Peter \\ Nee \\ Electrical Energy Conversion (E2C) Laboratory \\ School of Electrical Engineering, \\ KTH Royal Institute of Technology, \\ Stockholm, Sweden \\ rabkow@kth.se
}

\author{
Mariusz Zdanowski \\ Institute of Control and Industrial Electronics \\ Warsaw University of Technology \\ Warsaw, Poland
}

\begin{abstract}
The paper proposes a novel topology of a simple base drive unit for silicon carbide bipolar junction transistors (BJTs) based on the current-source principle. Energy stored in a small, air-cored inductor is employed to generate a current peak forcing the BJT to turn-on (10-20ns) very rapidly. The driver enables very high switching performance and very low switching losses of the driven BJT. Both the current source and the unit delivering the steady-state current to the base are supplied from the same low-voltage source in order to limit power consumption. Operation principles as well as selected design issues are discussed in the paper and illustrated by experiments. The $1200 \mathrm{~V} / 6 \mathrm{~A} \mathrm{SiC} \mathrm{BJT}$ driven by the proposed circuit shows a very fast switching speed.
\end{abstract}

Keywords-Silicon carbide; bipolar transistor; base drive unit, current source

\section{INTRODUCTION}

Silicon Carbide ( $\mathrm{SiC}$ ) power electronics has been in a kind of waiting-mode for approximately two decades [1]-[5]. The main reason to this is that the material itself has not been sufficiently mature for series production of power semiconductor components. During recent years, however, tremendous improvements in material quality have been achieved, and as a result several power semiconductor components are now available on the market. One of the most promising active switches in $\mathrm{SiC}$ is the bipolar junction transistor (BJT) which offers very low on-state voltage drops and very high switching speeds. These properties make the SiC BJT a very interesting switch candidate for a multitude of applications ranging from switch-mode power supplies and photovoltaic converters to converters for hybrid electric vehicles or electric traction applications.

As switching speeds of the order of 20 ns have been reported for $\mathrm{SiC}$ BJTs [6] it is very tempting to apply these devices in dc-dc converters, where high switching frequencies can substantially reduce the volume and cost of passive components. In order to fully exploit the benefits of the high switching speed, however, both the main circuit and the base drive unit of the BJT have to be carefully designed for this purpose. As will be shown below, this is not an entirely trivial task.

The requirements for a $\mathrm{SiC} \mathrm{BJT}$ base drive unit are higher than for drivers of Si IGBTs, Si MOSFETs, as well as for SiC normally-on JFETs or SiC MOSFETs. Besides providing dynamic current peaks to turn-on/turn-off the device, the second function of the driver is to supply a base current during conduction period [6]. This allows the bipolar transistor to operate in deep saturation with low voltage drop across collector-emitter terminals. The need for a controlling current during the conduction is a property in common with the normally-off (enhancement-mode) SiC JFET. This means that the same kind of base-drive units can be used for the two types of devices, but the gate current of the JFET is lower than the base current for the BJT [7], [8], [9].

The first attempts to design $\mathrm{SiC}$ BJT drivers, applied to $\mathrm{SiC}$ BJTs with low current ratings, were based on state-of-the art IGBT/MOSFET driver components. A typical gate driver equipped with a speed-up capacitor (Fig. 1a) is able to drive SiC BJTs with turn-on and turn-off times counted in tens of nanoseconds according to the recommendations of the manufacturer [6]. The disadvantage of this solution is that optimum operation parameters are hard to find as high switching speed requires high supply voltage/capacitor values. In order to reach low power consumption, however, exactly opposite measures should be taken. One solution to this problem is presented in [10] where a dual-source (2SRC) driver is proposed. The authors split two functions of the base drive unit into two stages: a low-voltage stage to optimize power consumption caused by the static base current requirement, and a high-voltage stage capable of driving the BJT very rapidly (see Fig. 1b). With the 2 SRC design applied to a $2 \mathrm{~kW} \mathrm{dc/dc}$ boost converter an efficiency of $98.9 \%$ at $100 \mathrm{kHz}$ was measured while the power consumption of the driver was less than 1.6 W [10], [11]. Further evaluation of the 2SRC driver led to the conclusion that some features could be recognized as drawbacks.

- Two voltage sources $\left(\mathrm{V}_{\mathrm{CCH}}, \mathrm{V}_{\mathrm{CCL}}\right)$ make the design complicated, 


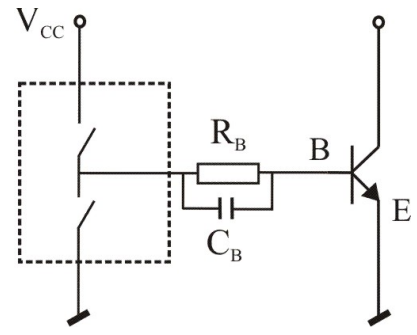

b)

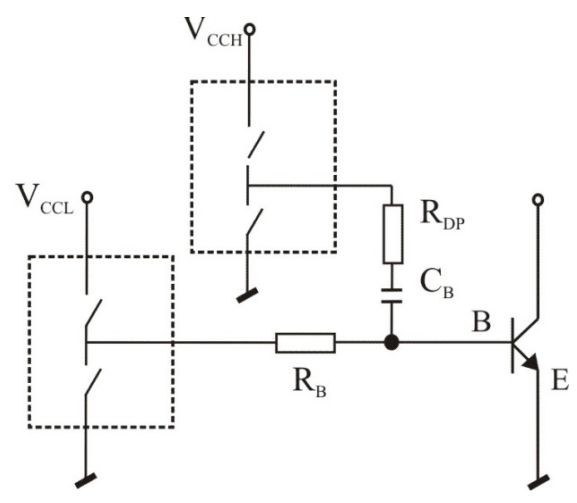

$\mathrm{c}$

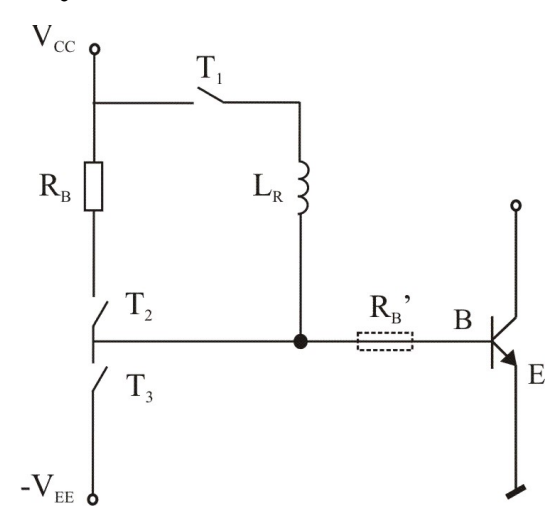

Fig. 1. SiC BJT base drivers: solution with speed-up capacitor (a) dual-source with resistors and capacitor (b) and proposed circuit with current source (c)

- The base capacitor $C_{B}$ is a source of high-frequency oscillations with parasitic inductances of the transistors package.

- The base capacitor must be discharged to make the driver ready for the next turn-on action and this is the reason to an associated duty-cycle limitation.

- The lack of a negative voltage supply could be a problem when devices operate in a leg configuration and an undesired turn-on may occur.

All mentioned problems were motivations to the search for a new solution to the BJT base drive problem - a solution offering the same or better performance than the 2SRC driver, but with a simpler design.

\section{CURRENT-SOURCE DRIVER IDEA}

A new current-source (CS) base-drive unit, proposed in this paper, is presented in Fig. 1c. The main difference, in comparison to the $2 \mathrm{SRC}$ driver, is that the part delivering the dynamic current at turn-on is supplied from the same lowvoltage source as the static part. This is possible thanks to the use of an inductor $L_{R}$ which is the key part of a simple current source [12]. The operation principles of the CS driver are illustrated in Fig. 2 where the control signals of three transistors and the current through the inductor $\mathrm{L}_{\mathrm{R}}$ as well as the base current can be seen.

The analysis starts when the driver control signal (IN) is low and the BJT is in the off-state. Switches $\mathrm{T}_{1}$ and $\mathrm{T}_{2}$ are off but $T_{3}$ is in the on-state providing negative bias. The rising edge of the control signal IN (observed at time $t_{A}$ ) turns on the switch $T_{1}$. The sum of the voltages $V_{C C}$ and $V_{E E}$ applied to the inductor $L_{R}$ leads to a linear increase of the current. At a certain instant when the inductor current has reached the desired peak value $I_{L R P}\left(t_{B}\right)$, the switch $\mathrm{T}_{3}$ is turned off. This breaks current path to the negative pole $V_{E E}$ and the inductor current is now conveyed into the base of the SiC BJT. Energy stored in $L_{R}$ is consumed to build up the current peak necessary to turn the transistor on. The turn-on of the upper switch $\mathrm{T}_{2}$ initiates the steady-state current delivered to the base from $V_{C C}$. Note, that the inductor current starts falling at $t_{B}$ as the applied voltage is reduced to merely the difference between $V_{C C}$ and $v_{B E}$. The rise time of the base current is limited by the time $t_{C}$, when the peak base current $\mathrm{I}_{\mathrm{BP}}$ is close to the inductor current. This means that most of the inductor energy is employed and switch $\mathrm{T}_{1}$ should be turned off at $t_{C}$. During the time between $t_{C}$ and $t_{D}$ the inductor current falls to zero and the base current reaches the fixed value $I_{B M}$ required for the collector current conduction.

The turn-off process starts with the falling edge of the input control signal IN $\left(t_{E}\right)$. Switches $\mathrm{T}_{2}$ and $\mathrm{T}_{3}$ are turned off and turned on respectively. The negative voltage $V_{E E}$ applied to the base-emitter terminals results in the dynamic current peak of the base current which turns off the SiC BJT. This process ends at $t_{F}$ when the base current equals zero again.

As the base resistor $R_{B}$, adjusting steady-state base current, is moved to the supply side, the dynamic current slope is limited only by the parasitic inductances and resistances. Thus, a very fast turn-on, usually expected from $\mathrm{SiC}$ transistor, can be easily achieved. The switching speed at turn-

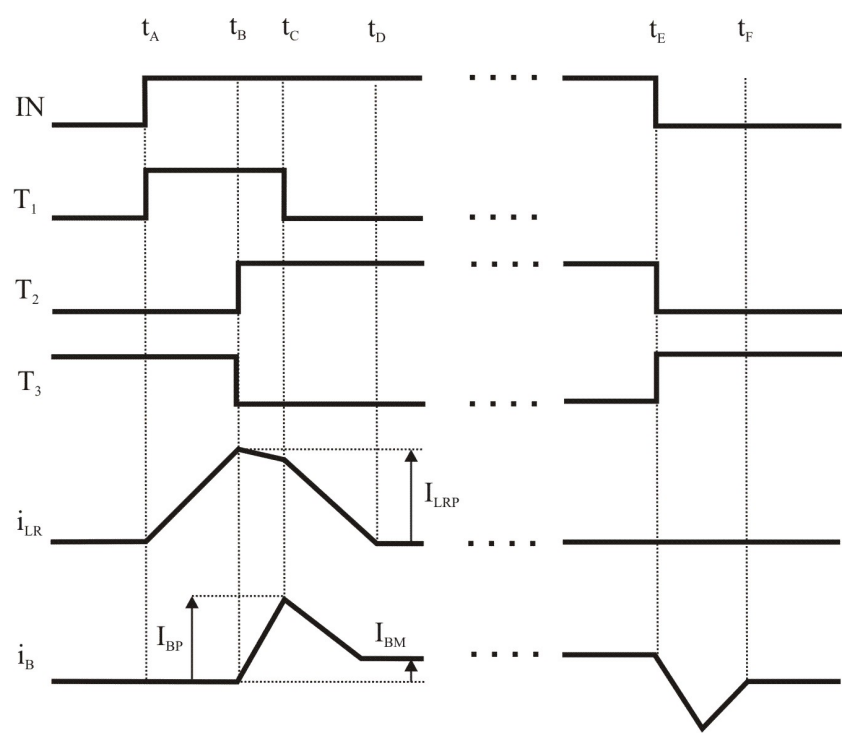

Fig. 2. Illustration of operation principles of the proposed current-source base driver 
on can be adjusted by means of $I_{L R P}$ by changing the rise times $t_{R}$ (between $t_{A}, t_{B}$, and $t_{C}$ ) or the value of $L_{R}$. Both the turn-on and turn-off switching speeds may be controlled by application of an additional resistor $\mathrm{R}_{B}^{\prime}$ as illustrated with the dashed lines in Fig. 1c.

The application of the inductor as an energy buffer for current peaks limits the problem of ringing caused by parasitic inductances. Aiming for effective utilization of the inductor current to build up the base current peak, the value of $L_{R}$ should be one order of magnitude higher than the parasitic inductance of the package (TO-247).

In the solution presented in [12] for the MOSFET, the inductor stage is also applied to achieve a fast turn-off. This could obviously be established by adding more transistors to the schematic in Fig. 1c. This would, however, make the circuit more complex, not to mention the special signal sequence for the, at least five, transistors which would need additional logic circuits. Actually, the desired fast turn-off is possible using only the three transistors in the proposed circuit in combination with the negative supply voltage $V_{E E}$.

Another important difference between the solution in [12] and the proposed simple driver design is the lack of inductor energy regeneration. Actually, this feature is not required as the remaining energy of the inductor after turn-on is not wasted. After the turn-on process of the main transistor (the duration between $t_{B}$ and $t_{C}$ ) the decreasing inductor current supports the steady-state current of the base. All in all, the majority of the power consumption of the base-drive unit is associated with the steady-state base current and possible savings would be significantly lower than $10 \%$.

\section{PRACTICAL REALIZATION}

The proposed CS base driver circuit was designed to control a $1200 \mathrm{~V} / 6 \mathrm{~A} \mathrm{SiC} \mathrm{BJT} \mathrm{[13]} \mathrm{with} \mathrm{the} \mathrm{use} \mathrm{of} \mathrm{two} \mathrm{totem-}$ pole MOSFET drivers from IXYS (see schematic in Fig. 3a). The IXDD614 offers low on-state resistance, a peak current of $14 \mathrm{~A}$, and short nanosecond switching times [14]. A series diode (low-voltage Schottky) is added to the inductor $\mathrm{L}_{\mathrm{R}}$ to block the low-side transistor, complementary to $\mathrm{T}_{1}$.

Taking into account operation conditions (short current peaks), the inductor $L_{R}$ was designed with an air core. With this design the saturation current is high, but the simplicity was also important benefit. The number of turns was experimentally determined to be 15 while the diameter of the inductor was approximately $3 \mathrm{~mm}$. As can be seen in Fig. 4, the physical size of the inductor $L_{R}$ is comparable to other electronics parts of the circuit. The inductance value, less than $200 \mathrm{nH}$, was chosen to be one order of magnitude higher than the expected parasitic inductance of the TO-247 package. It is the opinion of the authors, that this type of inductor design may be more reliable and stable than the capacitors applied in the dynamic part of a comparable 2SRC driver [10].

Considering the $\mathrm{SiC} \mathrm{BJT}$ requirements regarding the steady-state base current the base resistor value was selected to be $5.6 \Omega$. The positive supply voltage was chosen to be $V_{C C}=5 \mathrm{~V}$ aiming for minimum power dissipation in the base resistor [10]. The negative supply voltage could also be a)

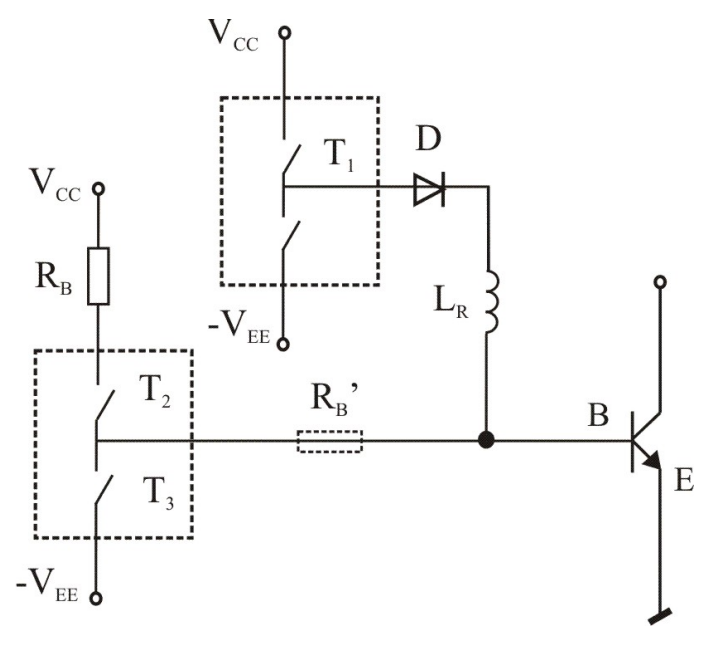

b)

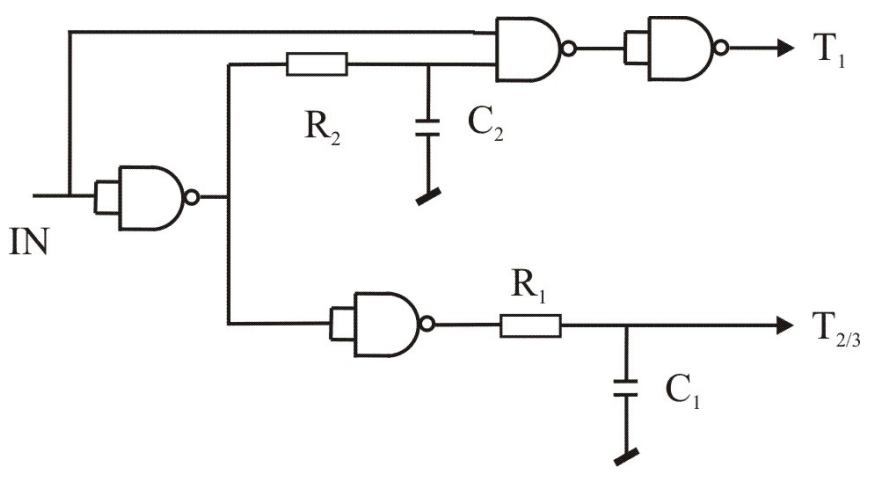

Fig. 3. Schematic of practical design of the simple current-source driver: power part (a) and logic circuit (b).

$V_{E E}=5 \mathrm{~V}$ in order to reduce the number of isolated $\mathrm{dc} / \mathrm{dc}$ converters, but in this design $V_{E E}=15 \mathrm{~V}$ was selected to ensure good dynamics at turn-on and turn-off. A base driver with more negative voltage is also less sensitive to noise when the $\mathrm{SiC} \mathrm{BJT}$ is operating in a bridge leg. Finally, two isolated dc/dc converters from TRACO (TMH2405S, TMH2415S) were employed to provide the positive and negative supplies.

The advantage of the proposed CS base-drive unit in reference to the solution presented in [12] is the simple control sequence which is merely constituted by a slightly modified input logic signal (see Fig. 2). As three switches $\mathrm{T}_{1}-\mathrm{T}_{3}$ are inside two totem-pole drivers (Fig.3a), only two control signals are required. Basically, the control signal of $\mathrm{T}_{1}$ (upper totem-pole) is a short high-state pulse triggered by the rising edge of the input signal IN (originating from an optocoupler). The second required signal for $T_{2} / T_{3}$ equals the signal IN with a delayed rising edge (Fig. 2a). The delay of the control signal $\mathrm{T}_{2} / \mathrm{T}_{3}$ and the width of the $\mathrm{T}_{1}$ pulse are expected to be adjustable.

Due this simple control sequence both totem-pole drivers could be controlled with the use of a simple logic circuit (see Fig. 3b). Only four 2-input NAND gates (single IC) and two 
$\mathrm{RC}$ delay circuits are employed. Changing the time constant $\mathrm{R}_{1} \mathrm{C}_{1}$, the duration between times $\mathrm{t}_{\mathrm{A}}$ and $\mathrm{t}_{\mathrm{B}}$ is changed (see Fig. 2). This means that the rise time of the current through $L_{R}$ can be adjusted. The second delay circuit $\mathrm{R}_{2} \mathrm{C}_{2}$ controls the $\mathrm{T}_{1}$ pulse width (between $t_{A}$ and $t_{C}$ ). Obviously, the $\mathrm{R}_{2} \mathrm{C}_{2}$ time constant should be longer than $\mathrm{R}_{1} \mathrm{C}_{1}$ to set the duration between $t_{B}$ and $t_{C}$, necessary to build up the peak of the base current as can be seen in the sequence presented in Fig. 2.

The operation of the logic circuit is illustrated in Fig. 5, where experimental waveforms of the input signal IN (output from optocoupler) and the two control signals of the totem-pole drivers $T_{2} / T_{3}$ and $T_{1}$ are presented. The experimental results in Fig. 5 are in agreement with the ideal waveforms shown in Fig. 2. Delay times between the input IN and control signals, caused by propagation delays of the applied NAND gates, are in the range of $100 \mathrm{~ns}$.

\section{BASE-CURRENT ADJUSTMENT}

The output from the proposed driver circuit, the base current waveform, can be adjusted to meet the BJT driving requirements. Current peaks at turn-on and turn-off influence the switching behavior, the slopes of the collector-emitter voltage and collector current. The steady-state base current keeps device in deep saturation where very low voltage drops are achievable.

As mentioned above, the steady-state value $\mathrm{I}_{\mathrm{BM}}$ can be chosen by means of $V_{C C}$ and $\mathrm{R}_{\mathrm{B}}$. Both values should be as low as possible to reduce the power loss. A more detailed discussion can be found in [10]. Considering a $1200 \mathrm{~V} / 6 \mathrm{~A}$ $\mathrm{SiC} \mathrm{BJT}$ with a built-in $\mathrm{BE}$ voltage in the range of $3 \mathrm{~V}, V_{C C}=5$ $\mathrm{V}$ and $\mathrm{R}_{\mathrm{B}}=5.6 \Omega$ were selected to obtain $I_{B M}=320 \mathrm{~mA}$.

The peak value of the current at turn-on $I_{B P}$ was determined, similarly, based on the experiences from the 2SRC driver presented in [10]. The required range of $I_{B P}$ is assumed to be up to $10 \mathrm{~A}$, but $5 \mathrm{~A}$ would be enough to switch the $\mathrm{SiC} \mathrm{BJT}$ rapidly. Considering for simplicity the case with $\mathrm{R}_{\mathrm{B}}{ }^{\prime}=0$, $V_{C C}+V_{E E}=20 \mathrm{~V}, \mathrm{~L}_{\mathrm{R}}<200 \mathrm{nH}, \mathrm{d} i_{L R} / \mathrm{d} t$ should be approximately $1 \mathrm{~A} / 10 \mathrm{~ns}(100 \mathrm{~A} / \mu \mathrm{s})$ - see Fig. 6. Then, the duration between $t_{A}$ and $t_{D}$ is expected to be shorter than $200 \mathrm{~ns}$, even for maximum peak. This value seems to be a good tradeoff between the duty-cycle limitation issue and the magnitudes of the supply voltage and inductance. The shape of the base current at turn-on is adjustable within this range with the use of the variable resistors $R_{1}$ and $R_{2}$.

The peak value of the inductor current $I_{L R P}$ should be set by $\mathrm{R}_{1}$ changes to be slightly higher than required $I_{B P}$ due to the negative slope of $i_{L R}$ after $t_{B}$ - see (see Fig.6). Then, using this method exactly the required value of $I_{B P}$ is reached at time $t_{C}$ when $i_{B}$ equals $i_{L R}$.

The next step in the procedure of the current peak setting is to choose the pulse width of $T_{1}$ by adjusting the value of $R_{2}$. As can be seen in the example presented in Fig. 6a, the duration between $t_{B}$ and $t_{C}$, necessary to increase the base current, is less than $1 / 2$ of the total $T_{1}$ pulse width (90 ns). This seems to be a fair ratio to obtain a suitable shape of the current peak. Another example (Fig. 6b) illustrates an

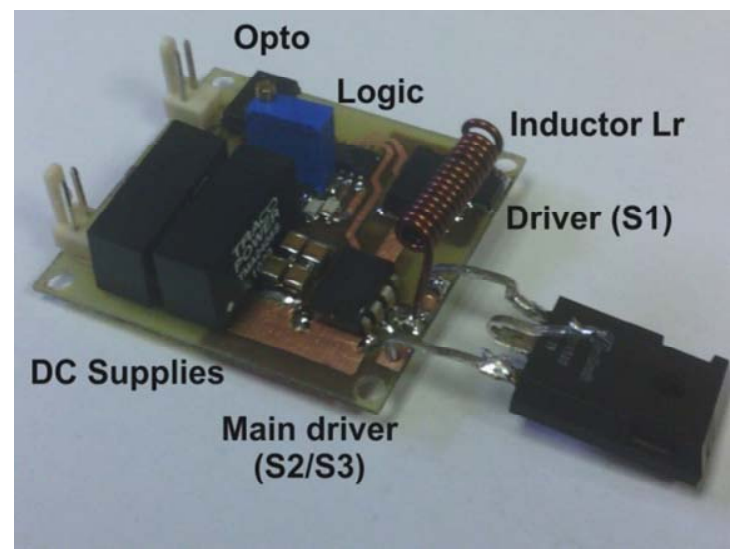

Fig.e 4. Photo of the PCB design $(45 \times 42 \mathrm{~mm})$ of the simple CS driver.

a)

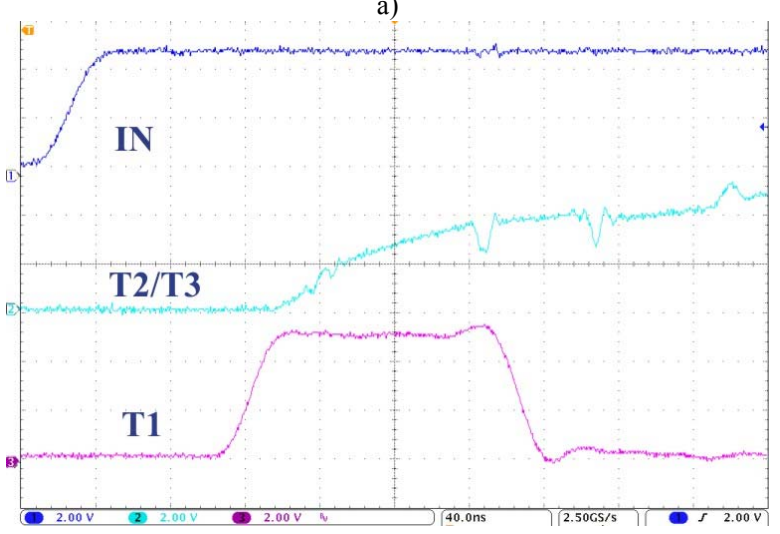

b)

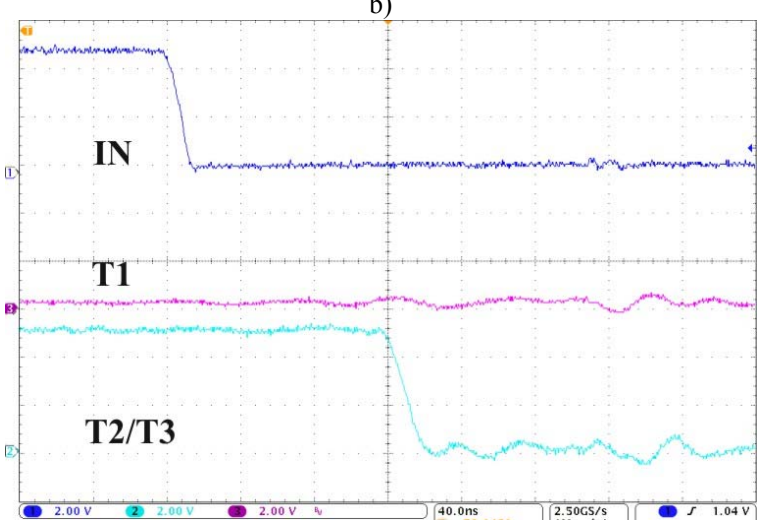

Fig. 5 Example of the control sequence - from the top: input signal, control signal of T1 and T2/T3 and T1 (all 2V/div, 40ns/div): a) turn-on, b)turn-off

extended $\mathrm{T}_{1}$ pulse $\left(\left(t_{C}-t_{B}\right)>50 \%\right)$ which increases the width of the base current. This results in increased RMS values of inductor and base currents and, consequently, higher power losses. Usually, the $6 \mathrm{~A} \mathrm{SiC} \mathrm{BJT}$ is turned on approximately 20-30 ns after $t_{B}$ (see Fig. 8) but for devices with bigger chip sizes extended pulses of the base current may be necessary.

The base current peak at turn-off can also be adjusted but not that precisely as at turn-on. Changing the values of $V_{E E}$ and $R_{B}^{\prime}$, the shape of the negative current peak can be set. Two examples of the base current at turn-off are presented in Fig. 7. Without the resistor $R_{B}^{\prime}$, when the rise of the current 


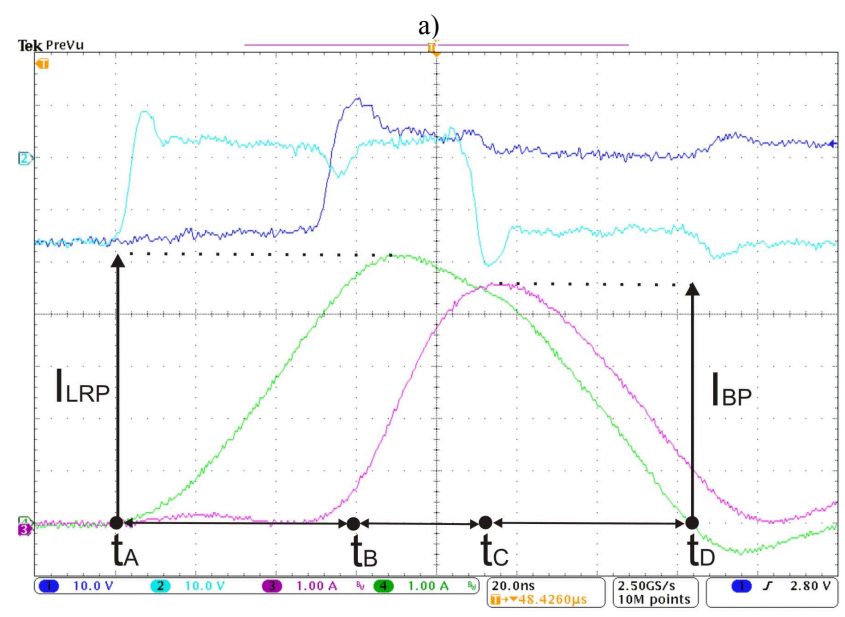

b)

13:Ar:2012

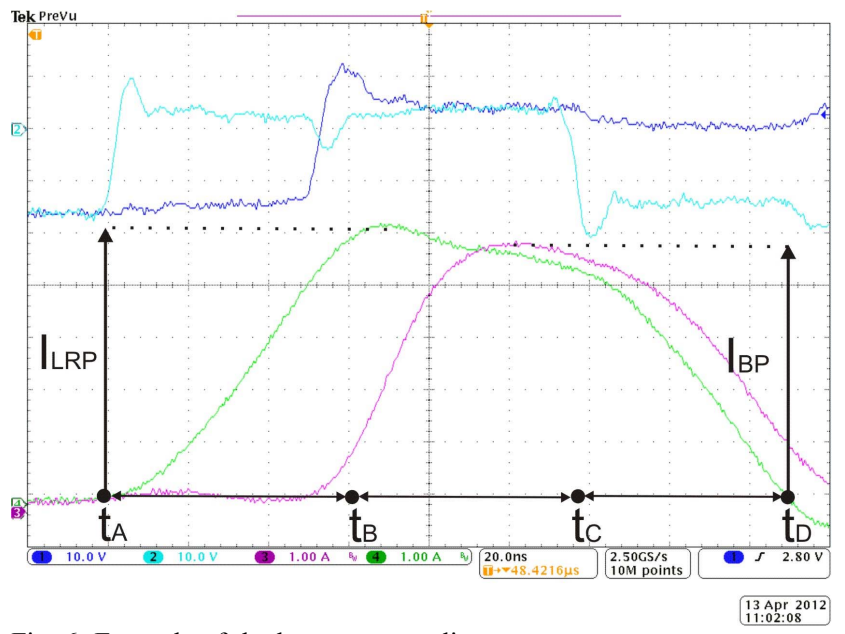

Fig. 6. Example of the base current adjustment at turn-on: correct current pulse (a) pulse with extended width (b). Waveforms: $T_{1}$ (light blue) and $\mathrm{T}_{2} / \mathrm{T}_{3}$ (dark blue) totem-pole output, inductor $\mathrm{L}_{\mathrm{R}}$ (green) and base (violet) currents. All voltages $10 \mathrm{~V} / \mathrm{div}$, currents $1 \mathrm{~A} / \mathrm{div}$, time scale $20 \mathrm{~ns} /$ div.

peak is limited only by the parasitic inductances and the totem-pole driver resistance, the maximum (negative) value $I_{B P_{-}}$is approximately $2 \mathrm{~A}$ (Fig. 7a). Application of $\mathrm{R}_{\mathrm{B}}^{\prime}=4.7 \Omega$ reduces this value by a factor two (Fig. $7 \mathrm{~b}$ ). The current oscillations visible in Fig. 7a are strongly damped.

\section{DOUBle-Pulse MeAsurements}

The model of the CS base drive unit was applied to control the $\mathrm{SiC} \mathrm{BJT}$ [13] tested in a standard double-pulse procedure. The 1200 V/6 A SiC BJT with 1200 V/5 A SiC Schottky were switched using a direct voltage of $600 \mathrm{~V}$ and nominal current. The voltage measurement was conducted with a high-voltage P5200 probe while two Rogowski coils (CWT mini) were employed to measure current.

The turn-on performance of the SiC BJT with CS driver is presented in Fig. 8 where base and collector currents and the collector-emitter voltage can be seen. The base current peak was set by changing $R_{B}^{\prime}$ from 0 to $4.7 \Omega$ while the value of the

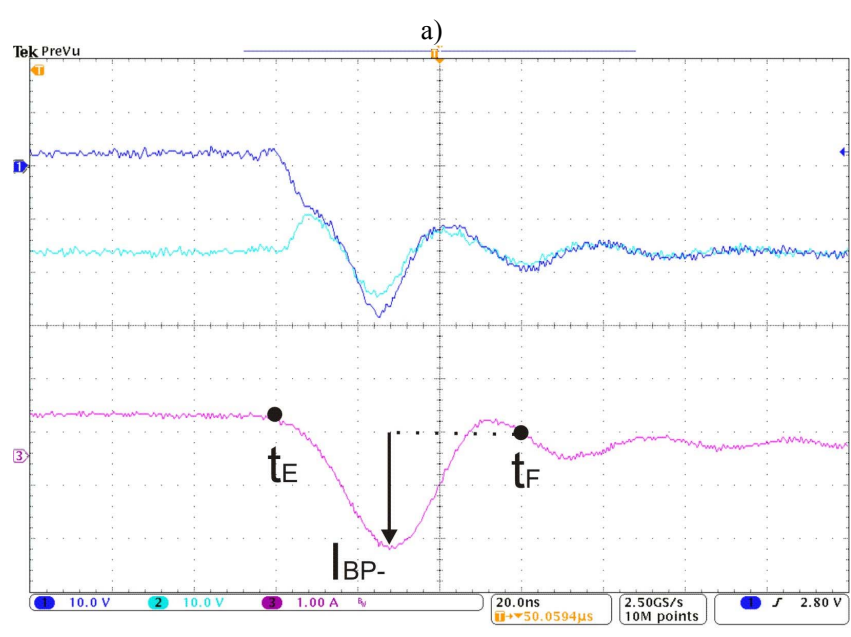

b)

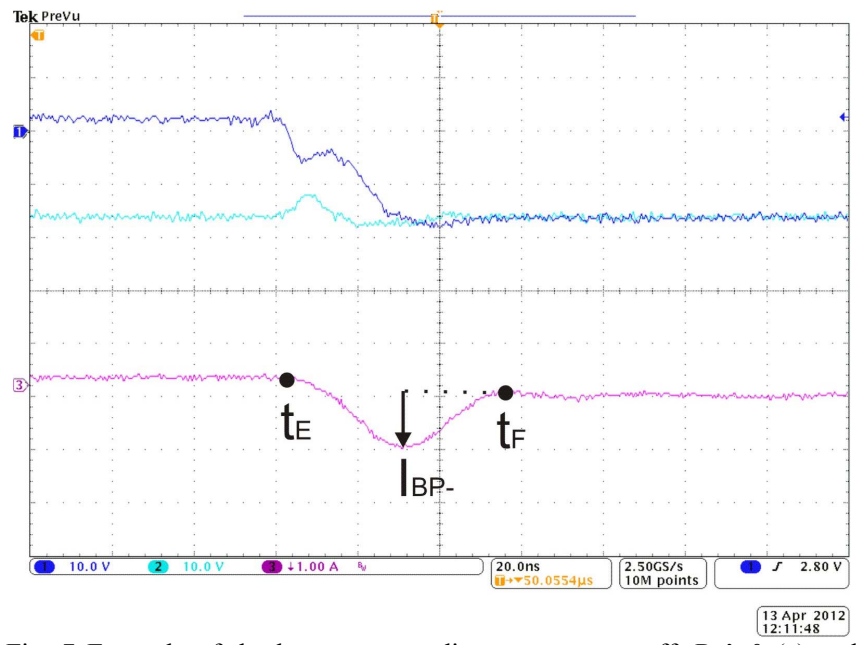

Fig. 7 Example of the base-current adjustment at turn-off: $R_{B}{ }^{\prime}=0$ (a) and $\mathrm{R}_{\mathrm{B}}{ }^{\prime}=4.7 \Omega$ (b). Waveforms: $\mathrm{T}_{1}$ (light blue) and $\mathrm{T}_{2} / \mathrm{T}_{3}$ (dark blue) totem-pole output and base current (violet) currents. All voltages $10 \mathrm{~V} / \mathrm{div}$, current $1 \mathrm{~A} / \mathrm{div}$, time scale $20 \mathrm{~ns} / \mathrm{div}$.

peak inductor current was constant. In result, the measured current peak reaches $10 \mathrm{~A}$ (Fig. 8a) and 7 A (Fig. 8b) respectively, which is lower value than observed when $V_{C E}=0 \mathrm{~V}$. The reason for being that could be an increased parasitic base-collector charge $\mathrm{Q}_{\mathrm{BC}}$ from 0 to $47 \mathrm{nC}$ with the switched voltage rise from 0 to $600 \mathrm{~V}$ [13]. Moreover, Rogowski coil which measures the base current is placed close to collector and emitter terminals due to a very limited space. It is also possible that the coil is influenced by flux generated by the collector/emitter current peaks.

The observed voltage slopes at turn-on are very fast: $15 \mathrm{~ns}$ and $25 \mathrm{~ns}$, corresponding to 40 (Fig. 8a) and $24 \mathrm{kV} / \mu \mathrm{s}$ (Fig. $8 b)$. A comparison of the two cases reveals that the measured collector current speed is rather influenced by parasitic inductances than by the driver circuit. In spite of some differences, both current slopes are measured to be approximately $100 \mathrm{~A} / \mu \mathrm{s}$. The waveforms suggest some parasitic capacitance in the circuit as the collector-emitter voltage starts falling before collector current reaches the 
a)

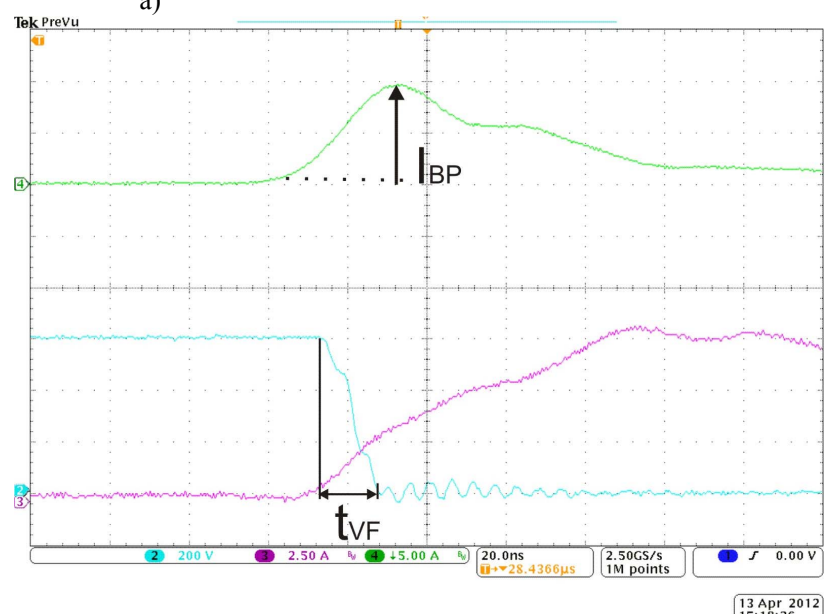

b)

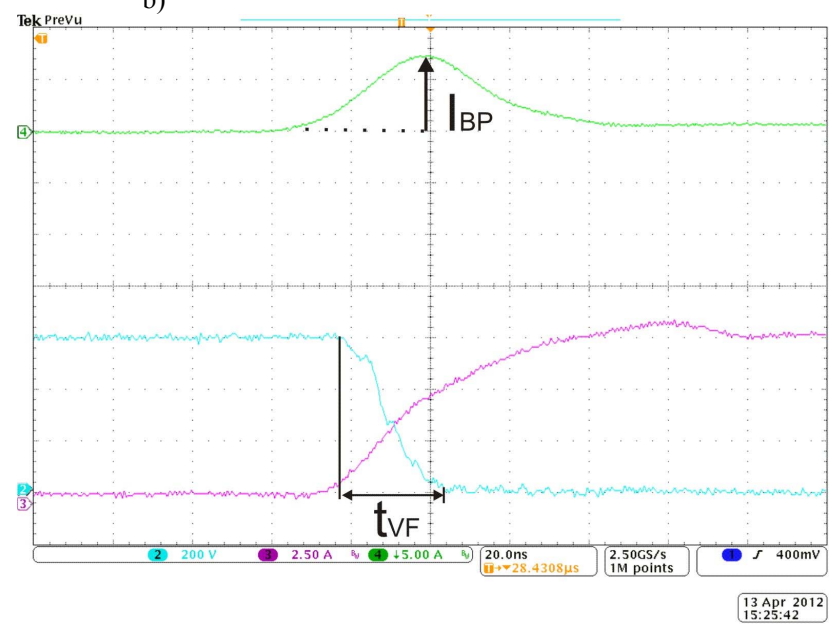

Fig. 8. SiC BJT turn-on process for two different values of $\mathrm{I}_{\mathrm{BP}}$ : $\mathrm{R}_{\mathrm{B}}{ }^{\prime}=0$ (a) and $R_{B}{ }^{\prime}=4.7 \Omega$ (b) (time scale $20 \mathrm{~ns} / \mathrm{div}$ ).

steady-state value. This means very low turn-on losses $\left(E_{O N}\right.$ equals 4 and $16 \mu \mathrm{J}$ for Fig. 8a and Fig. $8 \mathrm{~b}$ respectively), close to the soft turn-on case.

A similar, but opposite, phenomenon is observed during the $\mathrm{SiC}$ BJT turn-off presented in Fig. 9. The collector current contains a capacitive component which results in a current peak and increases the delay time before the current slope is falling. This time, the switching energy is increased and only slightly depends on the speed of the current and voltage slopes. The switching speed is again influenced by a change of $R_{B}$ ' from 0 to $4.7 \Omega$. The switching energy $E_{O F F}$ equals 118 and $135 \mu \mathrm{J}$ for Fig. 9a and Fig. 9b respectively. The voltage rise is again very fast. Values of 12 and 16 ns are observed (speed 50 and $37.5 \mathrm{kV} / \mu \mathrm{s})$.

Presented measurements prove that the proposed base driver is able to force the $\mathrm{SiC}$ BJT to switch very fast (low switching losses: total energy 122 and $151 \mu \mathrm{J}$ ) and to deliver a steady-state current to the base during the conduction period. Furthermore, adjustment of the switching speed is perfectly possible.

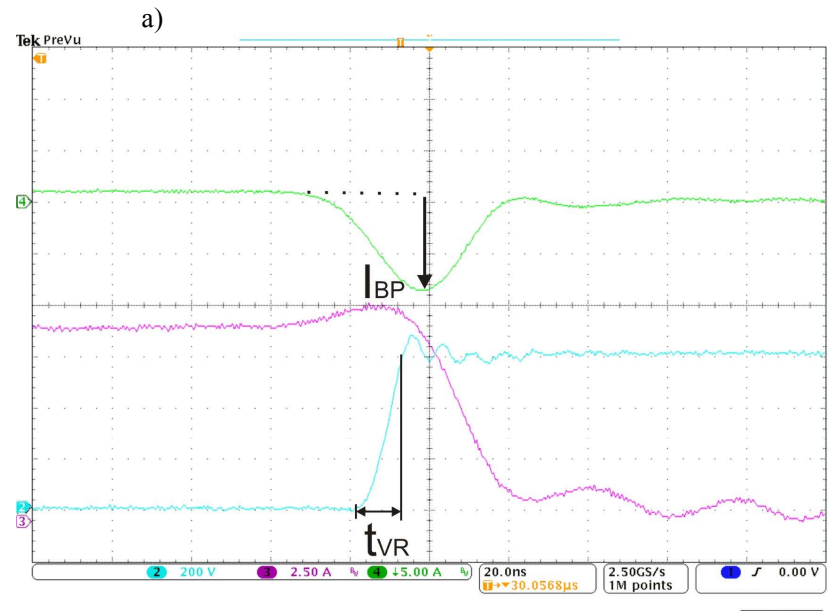

b)

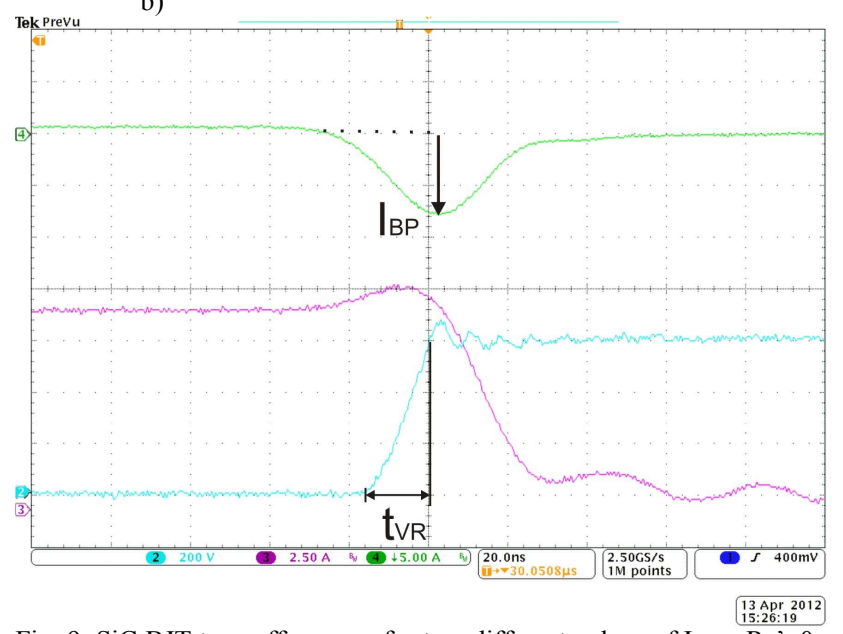

Fig. 9. SiC BJT turn-off process for two different values of $\mathrm{I}_{\mathrm{BP}(-)}: \mathrm{R}_{\mathrm{B}}{ }^{\prime}=0$ (a) and $R_{B}{ }^{\prime}=4.7 \Omega($ b) (time scale $20 \mathrm{~ns} /$ div).

\section{POWER CONSUMPTION}

A very important issue for the BJT base driver is the power consumption which depends on a number of parameters as supply voltage ranges, magnitude of the base current in the dynamic and steady-state, resistor and inductor values, frequency, and duty ratio. An initial test, conducted when the driver was operating at $100 \mathrm{kHz}$ with $50 \%$ duty ratio and steady-state/dynamic current equal to $320 \mathrm{~mA} / 2 \mathrm{~A}$ shows that the power consumption equals $1.9 \mathrm{~W}$ for $+5 /-15 \mathrm{~V}$ supply, $1.6 \mathrm{~W}$ for $+/-5 \mathrm{~V}$ supply, and $1.15 \mathrm{~W}$ for $+5 / 0 \mathrm{~V}$ supply, respectively. Obviously with the reduction of negative supply the current peaks are smaller but also logic circuitry (operating with ground connected to $-V_{E E}$ ) does not need a linear regulator. Another test shows that the driver consumes in off state $(-15 \mathrm{~V}) 767 \mathrm{~mW}$ with or without the transistor connected. The same test conducted for constantly on driver $(+5 \mathrm{~V})$ results in $540 \mathrm{~mW} / 2.4 \mathrm{~W}$ with $\mathrm{SiC}$ BJT. The next two series of measurements, presented in Fig. 10, were conducted for $+5 /-15 \mathrm{~V}$ supply, constant duty ratio $50 \%$, current peak $I_{B P}=5 \mathrm{~A}$ and various frequency values. The measured power losses, with or without main transistor, depend on frequency as more energy is required to generate current peaks. Most of the power loss is dissipated in the driver itself (1.1 W from 1.9 W@ 
$100 \mathrm{kHz}$ ) which is subject to future optimization. An estimation made on the base of various measurements shows that:

- parts of the driver operating at $+5 \mathrm{~V}$ level (TRACO supply, IXDDs, base resistor and $\mathrm{SiC}$ BJT) consume $1.1 \mathrm{~W}$

- $\quad$ parts of the driver operating at negative voltage (TRACO supply, linear regulator, logic circuit, IXDDs, SiC BJT) consume $0.5 \mathrm{~W}$

- inductor $\mathrm{L}_{\mathrm{R}}$, series diode and positive current peaks are responsible for $0.3 \mathrm{~W}$ of total power loss

while the $\mathrm{CS}$ driver is operating at $100 \mathrm{kHz}$ with duty ration $50 \%$. Accordingly, the proposed simple driver design has a power consumption that is slightly higher to the one of the 2SRC driver. On the other hand, the power loss distribution shows that optimization is very possible (i.e. by use $15 \mathrm{~V}$ logic and optocoupler).

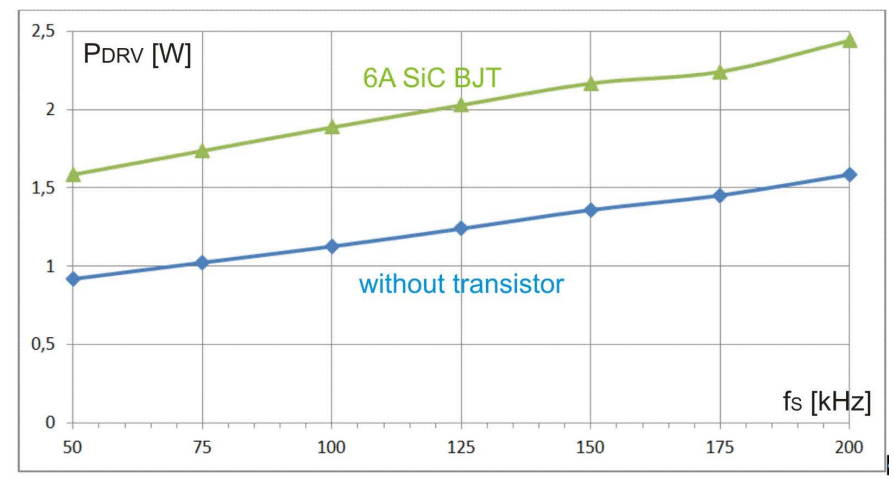

Fig. 10 Measured power consumption of the CS driver $(50 \%$ duty ratio, $5 \mathrm{~A}$ base current peak, $+5 /-15 \mathrm{~V}$ supply.

\section{CONCLUSIONS}

The paper proposes a novel topology of a base-drive unit for $\mathrm{SiC} \mathrm{BJTs}$ which employs the current-source principle to force the BJT to a very fast turn-on. The main advantage is that both parts of the driver: the steady-state and the dynamic are fed from the same low-voltage supply (i.e. $5 \mathrm{~V}$ ) which simplifies the design. The driver may be equipped with a negative supply voltage which would speed-up the turn-off process and keep the transistor in the off-state when used in a bridge-leg configuration. Both, speeds of turn-on and turn-off can be adjusted by circuit parameters.

The control sequence of the driver transistors cannot be described as complicated as only one additional, short pulse triggered by rising edge of input signal is required. That is why the logic circuitry is very simple and can be realized with four NAND gates and two RC circuits. The same can be said about power part of the driver: besides supply two totem-pole drivers with base resistor and light air-cored inductor are necessary.

The presented measurements show correct operation of the proposed driver: dynamic as well as static currents to the base are delivered as required. The power consumption, as was measured, is in the same range as for the 2SRC driver. Doublepulse tests performed to verify the switching performance of the $6 \mathrm{~A} \mathrm{SiC} \mathrm{BJT} \mathrm{confirmed} \mathrm{very} \mathrm{good} \mathrm{dynamic} \mathrm{properties,}$ especially the turn-on switching forced by the current source is very fast. This will lead, together with very low on-state losses of the BJT, to very low semiconductor power losses when the BJT operates in a power converter.

\section{REFERENCES}

[1] B. Ållebrand, H.-P. Nee, "On the possibility to use SiC JFETs in Power Electronic circuits", in Proc. Proceedings of the European Conference on Power Electronics and Applications (EPE), August 2001.

[2] Stephani D., "Status, prospects and commercialization of $\mathrm{SiC}$ power devices", Device Research Conference, 2001, p. 14.

[3] M. Bakowski, "Status and prospects $\mathrm{SiC}$ power devices," IEEJ Transactions on Industry Applications, vol. 126, No. 4, 2006, pp. 391399.

[4] Friedrichs, P.; "Silicon carbide power semiconductors - new opportunities for high efficiency," in Proc. 3rd IEEE Conference on Industrial Electronics and Applications, 2008. ICIEA 2008, vol., no., pp.1770-1774, 3-5 June 2008.

[5] N. Kaminski, State of the art and future of wide band-gap devices, in proc. Of $13^{\text {th }}$ European Conference on Power Electronics and Applications, EPE 2009.

[6] Drive circuit and switching, Application Note TSC-TR001, Transic, 2010.

[7] Ritenour, A., Sheridan, D.C., Bondarenko, V., Casady, J.B., "Saturation current improvement in $1200 \mathrm{~V}$ normally-off SiC VJFETs using nonuniform channel doping", 22nd International Symposium on Power Semiconductor Devices \& IC's (ISPSD), 2010, 361-364.

[8] Kelley, R., Ritenour, A., Sheridan, D., Casady, J., "Improved two-stage DC-coupled gate driver for enhancement-mode SiC JFET", TwentyFifth Annual IEEE Applied Power Electronics Conference and Exposition (APEC), 2010, 1838 - 1841.

[9] Wrzecionko B. et al., "Novel AC Coupled Driver for Ultra Fast Switching of Normally-off SiC JFETs", 36th Annual Industrial Electronics Society Conference IECON 2010, 605-612.

[10] J. Rabkowski, G. Tolstoy, D. Peftitsis, H.P. Nee, "Low-Loss HighPerformance Base-Drive Unit for SiC BJTs", IEEE Transactions on Power Electronics, Vol. 27, No. 5, May 2012, pp.2633-2643.

[11] Peftitsis D., Rabkowski J., Tolstoy G., Nee H.P., "Experimental comparison of a dc/dc converter with $\mathrm{SiC}$ JFETs and $\mathrm{SiC}$ bipolar transistors", $14^{\text {th }}$ European Conference on Power Electronics and Applications EPE'2011.

[12] Fu J., Zhang Z., Liu, Y.F., Sen, P., Ge, L., "A New High Efficiency Current Source Driver with Bipolar Gate Voltage", IEEE Transactions on Power Electronics, Vol. 27, Iss. 2, 2012, pp. 985-997.

[13] BitSiC1206BB-P1 datasheet, Transic, 2010.

[14] IXDD614 datasheet, Claire, 2011. 